$05,11,12$

\title{
Особенности конкуренции спиновой и псевдоспиновой подсистем в модельном купрате
}

\author{
(С) Ю.Д. Панов, В.А. Улитко, К.С. Будрин, Д.Н. Ясинская, А.А. Чиков \\ Уральский фредеральный университет, \\ Екатеринбург, Россия \\ E-mail: yuri.panov@urfu.ru
}

\begin{abstract}
Рассматрена конкуренция магнитного и зарядового упорядочения в модельном купрате в рамках упрощенной статической двумерной спин-псевдоспиновой модели. Эта модель эквивалентна двумерной разбавленной антиферромагнитной (AFM) модели Изинга с заряженными примесями. Представлены результаты среднего поля для исследуемой системы и их краткое сравнение с классическими расчетами Монте-Карло (МК). Численное моделирование показывает, что случаи сильного обмена и сильной зарядовой корреляции качественно различаются. Для сильного обмена AFM-фаза неустойчива по отношению к фазовому расслоению (ФР) на псевдоспиновую (зарядовую) и магнитную (спиновую) подсистемы, которые ведут себя как несмешивающиеся квантовые жидкости. Получено аналитическое выражение для температуры ФР.
\end{abstract}

Работа выполнена при поддержке Программы 211 Правительства Российской Федерации, соглашение 02.А03.21.0006 и проектов № 2277 и № 5719 Министерства образования и науки Российской Федерации и гранта РФФИ № 18-32-00837 18 .

DOI: 10.21883/FTT.2019.05.47574.03F

\section{1. Введение}

Одной из актуальных проблем в физике сверхпроводящих купратов является сосуществование и конкуренция спинового, сверхпроводящего и зарядового упорядочений. Изучение взаимосвязи магнетизма и сверхпроводимоста в купратах имеет долгую историю [1,2]. За последние пятнадцать лет появилось множество экспериментальных результатов, указывающих на наличие зарядового упорядочения [3-8] и взаимное влияние спинового и зарядового упорядочения в купратах [9-14]. В работе [15] была предложена модель, в которой уникальные свойства купратов связывались с неустойчивостью к переносу заряда состояний центров $\mathrm{CuO}_{4}$ в плоскостях $\mathrm{CuO}_{2}$. Для центров $\mathrm{CuO}_{4}$ в плоскости $\mathrm{CuO}_{2}$ это дает возможность рассматривать три многоэлектронных валентных состояния $\mathrm{CuO}_{4}^{5-, 6-, 7-}$ (соответствующих, формально, состояниям ионов меди $\left.\mathrm{Cu}^{1+, 2+, 3+}\right)$ как компоненты псевдоспинового триплета $S=1$ с $M_{S}=-1,0,+1$ соответственно и позволяет нам использовать псевдосиновый формализм для псевдоспина $S=1[15,16]$. Для рассмотрения конкуренции спинового и зарядового упорядочений в купратах была предложена упрощенная статическая двумерная спинпсевдоспиновая модель [17-19], являющаяся предельным случаем общей псевдоспиновой модели.

Гамильтониан статической спин-псевдоспиновой модели имеет вид

$$
\begin{aligned}
\mathscr{H}= & \Delta \sum_{i} S_{z i}^{2}+V \sum_{\langle i j\rangle} S_{z i} S_{z j}+\tilde{J} \sum_{\langle i j\rangle} \sigma_{z i} \sigma_{z j} \\
& -\tilde{h} \sum_{i} \sigma_{z i}-\mu \sum_{i} S_{z i},
\end{aligned}
$$

где $S_{z i}-z$-компонента псевдоспина $S=1$ на узле, $\sigma_{z i}=P_{0 i} s_{z i} / s-$ нормированная $z$-компонента спи- на $s=1 / 2$, умноженная на оператор проектирования $P_{0 i}=1-S_{i z}^{2}$. Параметры $\Delta=U / 2$ и $V>0$ определяют зарядовые корреляции на узле и между узлами решетки соответственно, $J=\tilde{J} / \mathrm{s}^{2}>0-$ обменное взаимодействие Изинга между ионами $\mathrm{Cu}^{2+}, h=\tilde{h} / s$ - внешнее магнитное поле, $\mu-$ химический потенциал, необходимый для учета условия постоянства допированного заряда, $n N=\sum\left\langle S_{z i}\right\rangle=$ const, где $n-$ плотность допированного заряда. Суммирование идет по узлам двумерной квадратной решетки, $\langle i j\rangle$ означает ближайших соседей. Эта спин-псевдоспиновая модель обобщает двумерную разбавленную антиферромагнитную (AFM) модель Изинга с заряженными примесями. В пределе $\Delta \rightarrow-\infty$ она сводится к модели Изинга для спина $S=1 / 2$ с фиксированной намагниченностью. При $\Delta>0$ результаты можно сравнить с моделью Блюма-Капеля [20,21] или с моделью Блюма-Эмери-Гриффитса [22]. Модель Изинга с подвижными заряженными примесями также была рассмотрена в работе [23]. Очевидно, что наиболее важным ограничением нашей модели для ее сравнения с реальными купратами является отсутствие в гамильтониане переноса заряда.

В работах $[17,18]$ были рассмотрены фазовые диаграммы основного состояния в приближении среднего поля. Было показано, что всего реализуются пять фаз основного состояния в двух различных пределах. В пределе слабого обмена, при $\tilde{J}<V$, все фазы основного состояния (COI, COII, COIII, FIM) соответствуют зарядовому упорядочению $(\mathrm{CO})$ типа шахматной доски при средней плотности заряда $n$. В то время, как в фазе COI нет спиновых центров $\left(\mathrm{Cu}^{2+}\right)$, фазы COII и COIII разбавляются невзаимодействующими спиновыми центрами, распределенными только в одной подрешетке. Такое ферримагнитное спиновое упорядочение является результатом приближения среднего поля, поэто- 
му численные расчеты классическим методом МонтеКарло (МК) показывают в этих случаях парамагнитный отклик при низких температурах. Фаза FIM также формально ферримагнитна. В этом случае спиновое AFM-упорядочение разбавляется невзаимодействующими зарядовыми центрами $\left(\mathrm{Cu}^{1+, 3+}\right)$, распределенными только в одной подрешетке. В пределе сильного обмена, при $\tilde{J}>V$, реализуются только фазы COI и AFM, в которых зарядовые центры равномерно распределены в обеих подрешетках.

Статья организована следующим образом. Мы представляем результаты расчета термодинамических свойств исследуемой системы в приближении среднего поля для и кратко сравниваем их с расчетами классическим методом МК в разд. 2. Расчеты МК показывают, что в пределе сильного обмена фаза AFM неустойчива по отношению к фазовому расслоению $(Ф Р)$ на подсистемы зарядовых и спиновых центров. В разд. 3 мы анализируем термодинамические свойства состояния ФР в рамках приближения сосуществования двух однородных фаз. Разд. 4 посвящен выводам.

\section{2. Приближение среднего поля}

В этом разделе кратко изложены результаты расчета термодинамических свойств в приближении среднего поля (СП). Мы используем неравенство Боголюбова для оценки большого потенциала $\Omega(\mathscr{H})$ : $\Omega(\mathscr{H}) \leq \Omega=\Omega\left(\mathscr{H}_{0}\right)+\left\langle\mathscr{H}-\mathscr{H}_{0}\right\rangle$. Стандартным образом мы вводим на квадратной решетке две подрешетки, $A$ и $B$, и выбираем

$$
\beta \mathscr{H}_{0}=\delta \sum_{i} S_{z i}^{2}-\sum_{\alpha, i_{\alpha}} \beta_{\alpha} S_{z i_{\alpha}}-\sum_{\alpha, i_{\alpha}} \gamma_{\alpha} \sigma_{z i_{\alpha}}
$$

где $\beta=1 / T, \delta=\beta \Delta, \beta_{\alpha}$ и $\gamma_{\alpha}-$ молекулярные поля, $\alpha=A, B$. Для оценки $\omega=\Omega / N$ получим выражение

$$
\begin{aligned}
2 \beta \omega & =\sum_{\alpha}\left[\left(\beta_{\alpha}-\xi\right) S_{\alpha}+\left(\gamma_{\alpha}-\eta\right) \sigma_{\alpha}\right. \\
& \left.-\ln 2\left(e^{-\delta} \cosh \beta_{\alpha}+\cosh \gamma_{\alpha}\right)\right]+z \nu S_{A} S_{B}+z i \sigma_{A} \sigma_{B},
\end{aligned}
$$

где $\xi=\beta \mu, v=\beta V, j=\beta \tilde{J}, \eta=\beta \tilde{h}, z=4-$ число ближайших соседей, и средние (псевдо)намагниченности для подрешеток $\left\langle S_{z i}\right\rangle_{\alpha}=S_{\alpha}$ и $\left\langle\sigma_{z}\right\rangle_{\alpha}=\sigma_{\alpha}$ имеют вид

$$
S_{\alpha}=\frac{\sinh \beta_{\alpha}}{\cosh \beta_{\alpha}+e^{\delta} \cosh \gamma_{\alpha}}, \quad \sigma_{\alpha}=\frac{\sinh \gamma_{\alpha}}{e^{-\delta} \cosh \beta_{\alpha}+\cosh \gamma_{\alpha}} .
$$

Минимизируя $\omega$ по $\beta_{\alpha}$ и $\gamma_{\alpha}$, получаем систему уравнений СП

$$
\beta_{\alpha}-\xi=-z \nu S_{\bar{\alpha}}, \quad \gamma_{\alpha}-\eta=-z j \sigma_{\bar{\alpha}},
$$

где $\bar{A}=B, \bar{B}=A$.

Выражения (5) должны быть дополнены зарядовым ограничением, $S_{A}+S_{B}=2 n$. Чтобы явно учесть это условие, можно ввести параметр зарядового порядка $a=\left(S_{A}-S_{B}\right) / 2$, и записать свободную энергию $f=\omega+\mu n$ как функцию $n, a$ и $\sigma_{\alpha}$ используя обратные соотношения для выражений (4):

$$
\begin{aligned}
e^{2 \beta_{\alpha}} & =\frac{\left(S_{\alpha} e^{\delta}+G_{\alpha}\right)^{2}-\sigma_{\alpha}^{2} e^{-2 \delta}}{\left(1-S_{\alpha}\right)^{2}-\sigma_{\alpha}^{2}}, \\
e^{2 \gamma_{\alpha}} & =\frac{\left(\sigma_{\alpha} e^{-\delta}+G_{\alpha}\right)^{2}-S_{\alpha}^{2} e^{2 \delta}}{\left(1-\sigma_{\alpha}\right)^{2}-S_{\alpha}^{2}},
\end{aligned}
$$

где

$$
G_{\alpha}=\left(1-S_{\alpha}^{2}-\sigma_{\alpha}^{2}+S_{\alpha}^{2} e^{2 \delta}+\sigma_{\alpha}^{2} e^{-2 \delta}\right)^{1 / 2} .
$$

Для неупорядоченного (NO) высокотемпературного решения при $h=0$ имеем $a=0, \sigma_{\alpha}=0$, и свободная энергия в расчете на узел примет вид

$$
\begin{aligned}
f_{\mathrm{NO}}= & \frac{z}{2} V n^{2}+\Delta|n|-\frac{1}{\beta} \ln \left(2 \frac{1+g_{0}}{1-n^{2}}\right) \\
& +\frac{|n|}{\beta} \ln \left(\frac{|n|+g_{0}}{1-|n|}\right),
\end{aligned}
$$

где $g_{0}=\left(\left(1-n^{2}\right) e^{-2 \delta}+n^{2}\right)^{1 / 2}$. Это позволяет нам вычислить все термодинамические функции NO-фазы. Энтропия, внутренняя энергия и удельная теплоемкость в расчете на узел имеют вид

$$
\begin{gathered}
s_{\mathrm{NO}}=\delta \frac{(1-|n|)\left(g_{0}-|n|\right.}{1+g_{0}}+\ln \left(2 \frac{1+g_{0}}{1-n^{2}}\right) \\
-|n| \ln \left(\frac{|n|+g_{0}}{1-|n|}\right), \\
e_{\mathrm{NO}}=\frac{z}{2} V n^{2}+\Delta \frac{n^{2}+g 0}{1+g_{0}}, \\
c_{\mathrm{NO}}=\delta^{2} \frac{\left(1-n^{2}\right)^{2} e^{-2 \delta}}{g_{0}\left(1+g_{0}\right)^{2}} .
\end{gathered}
$$

С точностью до независящего от температуры слагаемого $\frac{z}{2} V n^{2}$, выражения (7)-(10) соответствуют термодинамическим характеристикам идеальной системы невзаимодействующих псевдоспиновых (зарядовых) и спиновых дублетов, разделенных по энергии величиной $\Delta$. При $\Delta=0$, энтропия и внутренняя энергия становятся постоянными, поэтому теплоемкость равна нулю. Если $\Delta \neq 0$, теплоемкость имеет максимум при $T \propto|\Delta|$. В частности, если $n=0$,

$$
c_{\mathrm{NO}}=\left(\frac{\delta}{2}\right)^{2} \cosh ^{-2} \frac{\delta}{2}
$$

и максимум находится в точке $T=|\Delta| /(2 x)$, где $x$ является корнем уравнения $x=\operatorname{coth} x$.

Также можно написать явный вид магнитной восприимчивости при $h=0$ в NO-фазе. Полагая $S_{A}=S_{B}=n$ и $\sigma_{A}=\sigma_{B}=\sigma$ при $h \neq 0$, исключим $\xi$ из системы (5) и получим уравнение

$$
\sigma=\psi(\eta-x j \sigma, n),
$$


где введены следующие обозначения

$$
\begin{gathered}
\psi(x, n)=\frac{\left(1-n^{2}\right) \sinh x}{\cosh x+g(x, n)}, \\
g(x, n)=\left(\left(1-n^{2}\right) e^{-2 \delta}+n^{2} \cosh ^{2} x\right)^{1 / 2}, \\
g(0, n)=g_{0} .
\end{gathered}
$$

После стандартных вычислений получим

$$
\begin{gathered}
\left.\chi_{\mathrm{NO}}\right|_{h=0}=\left.\beta s^{2} \frac{\partial \sigma}{\partial \eta}\right|_{\eta=0}=s^{2} \frac{\chi_{0}(n)}{1+z \tilde{J} \chi_{0}(n)}, \\
\chi_{0}(n)=\beta \frac{1-n^{2}}{1+g_{0}},
\end{gathered}
$$

где $\chi_{0}(n)$ - нормированная восприимчивость в нулевом внешнем поле для идеальной системы невзаимодействующих псевдоспиновых и спиновых дублетов. Система уравнений (5) имеет ферримагнитные решения с $\sigma_{A}+\sigma_{B} \neq 0$ при $h=0$ [17], которые являются следствием приближения СП и не возникают в МК-расчетах. Из-за ограничения радиуса обменного взаимодействия в нашей модели ближайшими соседями, эти решения могут проявляться при численном моделировании как смесь антиферромагнитной и парамагнитной фаз. Недооценка парамагнитного отклика является в этих случаях систематической ошибкой метода СП. В дальнейшем мы будем рассматривать только AFМ-типы решений с $\sigma_{A}=-\sigma_{B}=\sigma$ при $h=0$. В этом случае, согласно уравнениям (5), выполняется соотношение $\gamma_{A}=-\gamma_{B}$. Намагниченности подрешеток $\sigma_{\alpha}$ являются монотонными функциями молекулярных полей $\gamma_{\alpha}$ в соответствии с уравнениями (4), следовательно, возможен только случай $\beta_{A}= \pm \beta_{B}$ для $\sigma \neq 0$. Это означает, что если $n \neq 0$, то существуют только АFМ-решения с $a=0, \sigma \neq 0$ и СО-решения с $a \neq 0, \sigma=0$. Случай $n=0$ следует рассматривать отдельно, поскольку он дает возможность для фрустрированных состояний, когда решения типа CO- и AFM-становятся вырожденными.

Термодинамические свойства фаз AFM- и CO-предполагают знание корней для уравнений (5) и могут быть рассчитаны численно. Кроме того, аналитически можно найти уравнения для температур фазовых переходов второго рода типа беспорядок-порядок и критических точек.

Для фазы АFM воспользуемся условием $\partial^{2} f / \partial \sigma^{2}=0$ при $\sigma=0$ что дает

$$
\left.\frac{\partial \gamma_{\alpha}}{\partial \sigma_{\alpha}}\right|_{\sigma_{\alpha}=0}=z j
$$

С учетом уравнений (6), получаем уравнение для температуры перехода $\mathrm{NO}-\mathrm{AFM}$

$$
\left(1-n^{2}\right) z j=1+g_{0}
$$

В частности, для $\Delta \rightarrow+\infty$ мы получим

$$
T_{\mathrm{AFM}}=(1-|n|) z \tilde{J}
$$

что совпадает с результатами работы [22]. Подставляя выражение (17) в (15) мы находим восприимчивость в точке перехода, $\chi_{\mathrm{NO}}=s^{2} /(2 z \tilde{J})$.
Для нахождения критической точки, которая разделяет переходы первого и второго рода, мы используем уравнение $\partial^{4} f / \partial \sigma_{4}=0$ на кривой сосуществования. После некоторых манипуляций получим

$$
g_{0}^{2}-2 g_{0}-3 n^{2}=0 .
$$

С учетом уравнения (17) мы получаем положение критической точки

$$
\begin{gathered}
T_{c 1}=z \tilde{J} \frac{1-n^{2}}{2+\sqrt{1+3 n^{2}}}, \\
\frac{\Delta_{c 1}}{T_{c 1}}=\frac{1}{2} \ln \frac{1-n^{2}}{1\left(1+n^{2}+\sqrt{1+3 n^{2}}\right)} .
\end{gathered}
$$

В частности, для $n=0, T_{c 1}=z \tilde{J} / 3, \Delta_{c 1} / T_{c 1}=-\ln 2$, что согласуется с результатами работы [22].

Восприимчивость в нулевом поле в AFM-фазе имеет вид

$$
\left.\chi_{\mathrm{AFM}}\right|_{h=0}=s^{2} \frac{\beta \psi^{\prime}(z j \sigma, n)}{1+z j \psi^{\prime}(z j \sigma, n)},
$$

где

$$
\psi^{\prime}(x, n)=\frac{\left(1-n^{2}\right)\left(g(x, n)+g_{0}^{2} \cosh x\right)}{g(x, n)(\cosh x+g(x, n))^{2}} .
$$

Параметр порядка AFM-фазы $\sigma$ при $h=0$ можно найти из уравнения

$$
\sigma=\psi(z j \sigma, n) \text {. }
$$

Аналогично, для фазы СО условие $\partial^{2} f / \partial a^{2}=0$ при $a=0$ дает

$$
\left.\frac{1}{2}\left(\frac{\partial \gamma_{A}}{\partial a}-\frac{\partial \gamma_{B}}{\partial a}\right)\right|_{\sigma_{\alpha}=0}=z v
$$

и для температуры перехода $\mathrm{NO}-\mathrm{CO}$ получаем уравнение

$$
\left(1-n^{2}\right) z v=1+g_{0}^{-1}
$$

В частности, для $\Delta \rightarrow-\infty$ получим

$$
T_{\mathrm{CO}}=\left(1-n^{2}\right) z V
$$

Уравнение для критической точки в фазе СО является более сложным

$$
2\left(1+3 n^{2}\right) g_{0}^{3}-g_{0}^{2}-6 n^{2} g_{0}+3 n^{2}=0,
$$

но при $n=0$ это дает $T_{c 2}=z V / 3, \Delta_{c 2} / T_{c 2}=\ln 2$.

Восприимчивость в нулевом поле в СО-фазе имеет вид

$$
\left.\chi_{\mathrm{CO}}\right|_{h=0}=s^{2} \frac{\frac{1}{2}\left(\chi_{0}(n+a)+\chi_{0}(n-a)\right)}{1+\frac{1}{2} z \tilde{J}\left(\chi_{0}(m+a)+\chi_{0}(n-a)\right)},
$$

где параметр порядка СО-фазы удовлетворяет уравнению

$$
a=\frac{1}{2 z v} \ln \left(\frac{(n+a+g(0, n+a))(1-n+a)}{(n-a+g(0, n-a))(1-n-a)}\right) .
$$



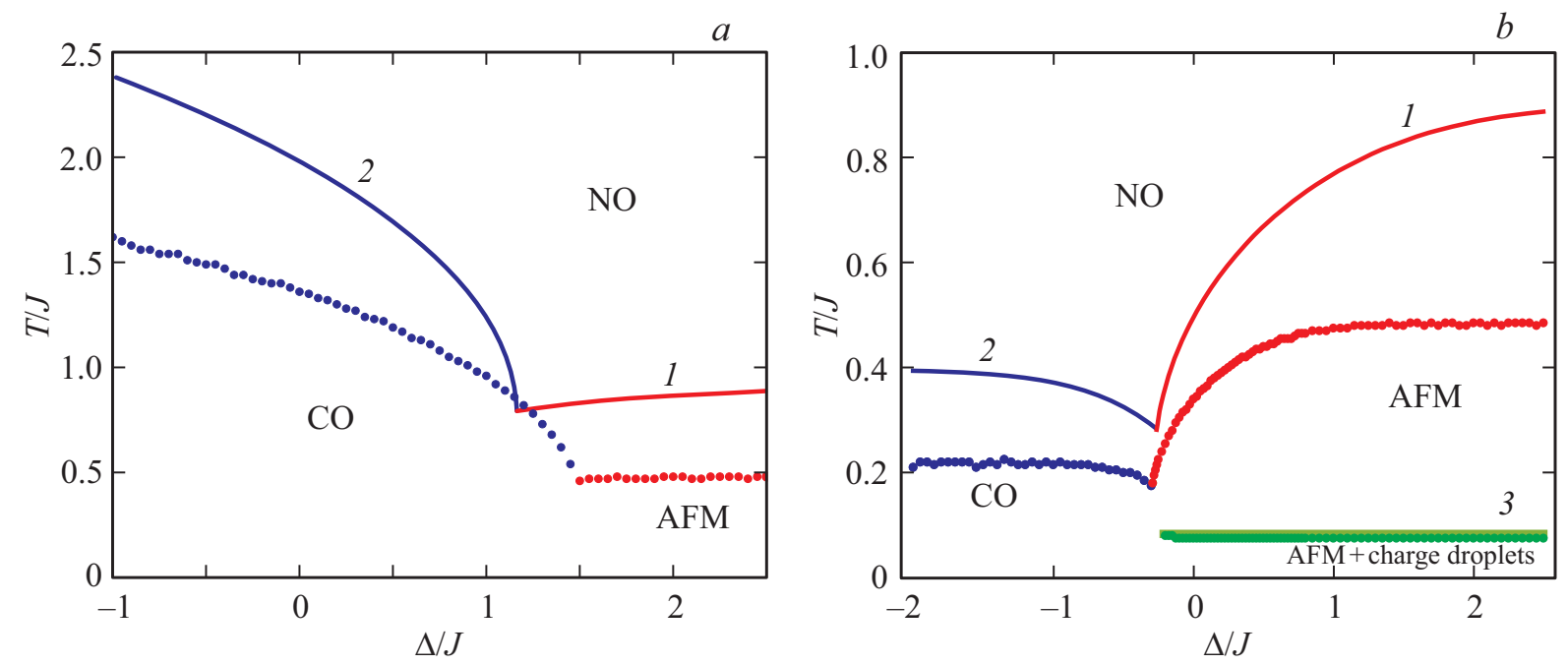

Рис. 1. Левая панель: случай слабого обмена: $n=0.1, \tilde{J}=0.25, V=1 ; b-$ случай сильного обмена: $n=0.1, \tilde{J}=0.25, V=0.1$. Точки соответствуют критическим температурам МК. На линиях 1-3 показаны значения критической температуры СП, заданной выражениями (17), (25) и (34).
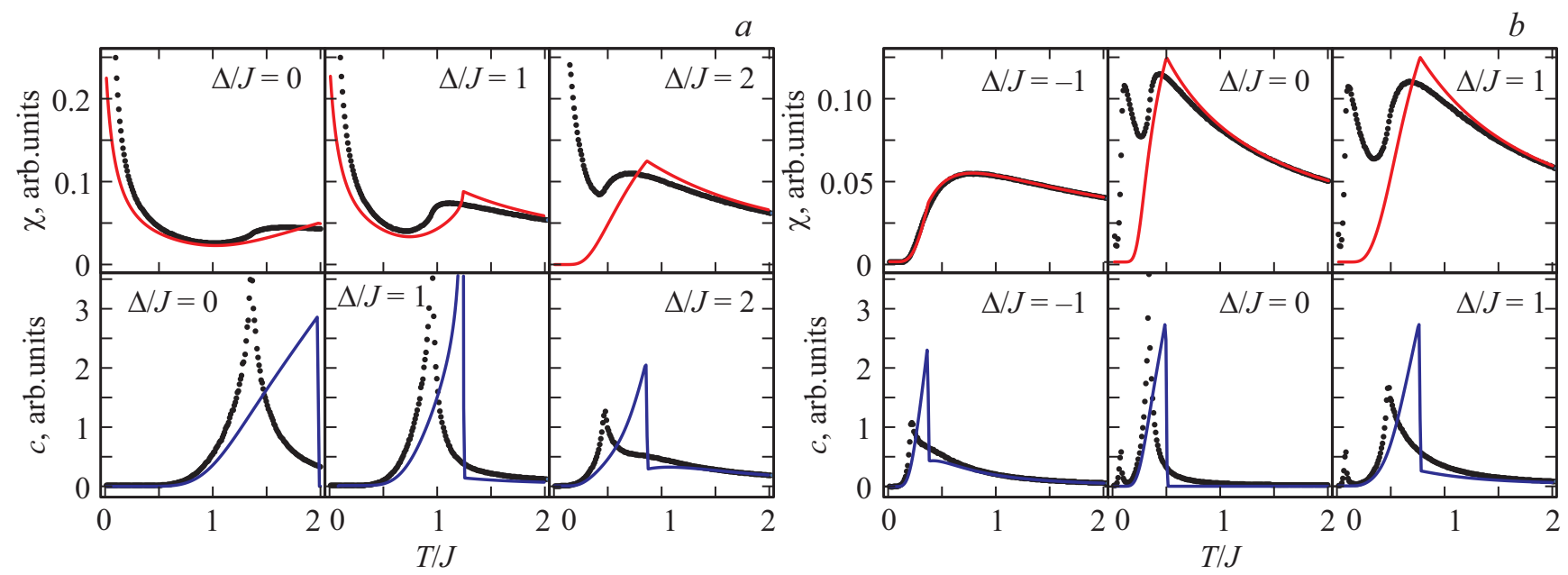

Рис. 2. Восприимчивость и удельная теплоемкость, полученные в приближении СП (сплошные линии) и методом МК (точки). $a-$ случай слабого обмена: $n=0.1, \tilde{J}=0.25, V=1 ; b-$ случай сильного обмена: $n=0.1, \tilde{J}=0.25, V=0.1$.

Общая формула для восприимчивости в нулевом поле, объединяющая случаи фаз $\mathrm{NO}$, AFM и $\mathrm{CO}$, дается формулой

$$
\chi=s^{2} \frac{\frac{1}{2} \beta\left(\psi^{\prime}(z j \sigma, n+a)+\psi^{\prime}(z j \sigma, n-a)\right)}{1+\frac{1}{2} z j\left(\psi^{\prime}(z j \sigma, n+a)\left(+\psi^{\prime}(z j \sigma, n-a)\right)\right.},
$$

где $\sigma$ - параметр порядка AFM-фазы, $a-$ параметр порядка СО-фазы.

Для численного моделирования был реализован высокопроизводительный алгоритм параллельных вычислений с использованием классического метода МК. Результаты расчетов методом МК показаны на рис. 1. Положение пика на температурной зависимости теплоемкости приблизительно (из-за конечного размера системы) соответствует температуре перехода беспорядок-порядок. Эти значения показаны точками. Температуры перехода в приближении СП (17) и (25) показаны сплошными линиями. Рис. 1 наглядно демонстрирует типичную, чуть менее чем в два раза, переоценку значения критической температуры в приближении СП. Зависимость от $\mathrm{n}$ критической температуры AFM-перехода в приближении СП показана ниже на рис. 3. Качественно ее вид согласуется результатами, полученными в приближении Бете в работе [23].

На рис. 2 мы сравниваем результаты для восприимчивости и теплоемкости, полученные в приближении СП и методом МК. Аналитические СП-зависимости показывают качественное согласие с результатами численного моделирования, и, в некоторых случаях, даже количественное совпадение результатов для области высоких температур. Основные расхождения вызваны различием в критической температуре и систематическими неточностями приближения СП для описания 


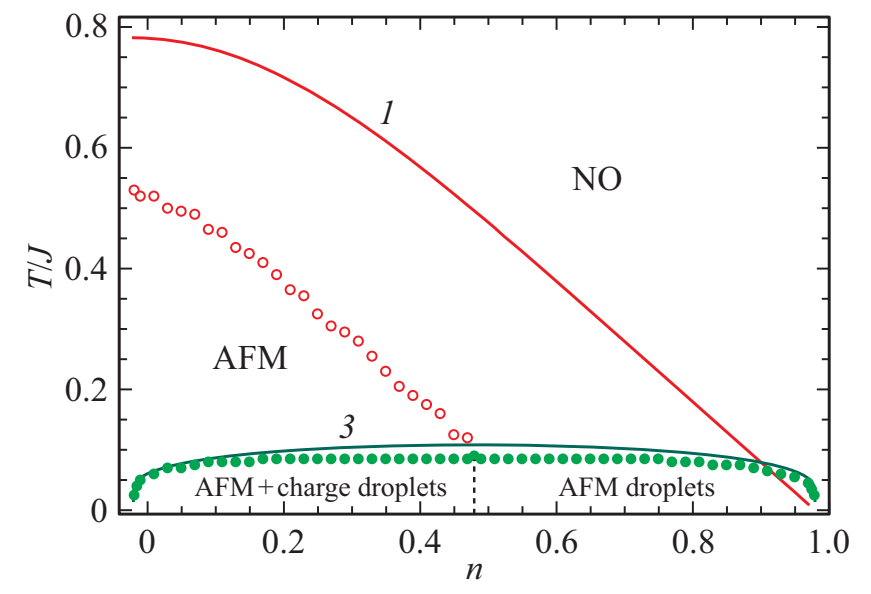

Рис. 3. Светлые кружки соответствуют максимумам восприимчивости при AFM-упорядочении, темные кружки - максимумам удельной теплоемкости при ФР, полученным методом МК. Значения параметров модели: $\Delta=1, \tilde{J}=0.25, V=0.14$. Линии 1 и 3 показывают значения критической температуры, заданные выражениями (17) и (34).

критических флуктуаций и парамагнитного отклика при низких температурах.

\section{3. Критическая температура фазового расслоения}

Согласно результатам численного моделирования классическим методом МК, температурные зависимости теплоемкости в пределе сильного обмена при положительном $\Delta$ демонстрируют два последовательных перехода. Непосредственное исследование состояния системы показывает, что первым переходом является AFM-упорядочение. При дальнейшем понижении температуры, в спиновой подсистеме, представляющей собой AFM-матрицу, разбавленную случайно распределенными заряженными примесями, происходит конденсация примесей в зарядовые капли. Другими словами, разбавленная AFM-фаза в пределе сильного обмена неустойчива по отношению к макроскопическому фазовому расслоению (ФР) на псевдоспиновую (зарядовую) и магнитную (спиновую) подсистемы. На этом этапе AFM-матрица выталкивает заряженные немагнитные примеси для минимизации поверхностной энергии. Отметим, что в пределе слабого обмена заряженные примеси распределены случайным образом по матрице АФМ вплоть до $T=0$, и также, заряженные примеси беспорядочно распределяются в СО-фазе, так как в приближении взаимодействия только ближайших соседей энергии всех возможных распределений дополнительных зарядов по СО-матрице равны. Результаты нашего численного моделирования аналогичны результатам, полученным для бинарных сплавов в работах [24,25].

Для описания термодинамических свойств неоднородного состояния мы используем модель, разработанную в работах [26-28] для макроскопического ФР в электронных системах. Эта модель основана на построении Максвелла. Предполагая сосуществование двух макроскопических однородных фаз, обозначенных как 1 и 2, запишем свободную энергию ФР-состояния в расчете на узел в виде

$$
f_{\mathrm{PS}}=m f_{1}\left(n_{1}\right)+(1-m) f_{2}\left(n_{2}\right),
$$

где $m$ - доля системы с плотностью $n_{1}, 1-m-$ доля системы с плотностью $n_{2}$, так, что $m n_{1}+(1-m) n_{2}=n$. В нашем случае одна фаза состоит из зарядовых центров (C), а другой - спиновая AFM-фаза без примесей, следовательно $n_{1}=\operatorname{sgn} n, n_{2}=0$ и $m=|n|$. Точка перехода определяется уравнением

$$
|n| f_{C}(1)+(1-|n|) f_{\mathrm{AFM}}(0)=f_{\mathrm{AFM}}(n) .
$$

Свободная энергия зарядовых центров $f_{C}(1)=2 V+\Delta$. Свободную энергию AFM-фазы можно записать в виде

$$
\begin{aligned}
f_{\mathrm{AFM}}(n)= & \frac{z}{2}\left(V n^{2}+\tilde{J} \sigma^{2}\right)+|n| \Delta \\
& -\frac{1}{\beta} \ln \left(2 \frac{\cosh (z j \sigma)+g(z j \sigma, n)}{1-n^{2}}\right) \\
& +\frac{|n|}{\beta} \ln \left(\frac{|n| \cosh (z j \sigma)+g(z j \sigma, n)}{1-|n|}\right) .
\end{aligned}
$$

Мы предполагаем, что $\Delta>0$ и рассматриваем случай низких температур, так $\delta \gg 1$ и $j \gg 1$. В этом приближении, с учетом уравнения (23), мы получим $|\sigma|=1-|n|$. Окончательно, уравнение (32) дает следующее выражение для температуры ФР

$$
T_{\mathrm{PS}}=\frac{|n|(1-|n|)}{|n| \ln |n|+(1-|n|) \ln (1-|n|)} \frac{z(V-\tilde{J})}{2} .
$$

Это выражение не зависит от $\Delta$ что согласуется с результатами МК для $T_{\mathrm{PS}}$ на рис. 1 .

Концентрационные зависимости критических температур для AFM-перехода и ФР показаны на рис. 3. Кружки обозначают результаты МК для максимумов восприимчивости при AFM-упорядочении, а точки показывают максимумы теплоемкости при ФР. Сплошная линия 3 показывает температуру ФР, определяемую формулой (34). Эта температура согласуется с результатами МК неожиданно хорошо, тогда как зависимость в приближении СП (линия 2) для критической температуры АFМ-упорядочения, определяемая формулой (17), становится качественно неверной при $|n|>0.5$. Отметим также, что выражение (34) для температуры ФР, основанное на построении Максвелла, дает более низкие значения по сравнению с найденными в работе [22].

\section{4. Заключение}

Мы рассмотрели статическую двумерную спинпсевдоспиновую модель на квадратной решетке, которая обобщает разбавленную антиферромагнитную модель Изинга. Мы сравнили аналитические результаты 
в приближении СП с результатами численного моделирования классическим методом МК. Анализ удельной теплоемкости и восприимчивости, полученных методом МК, показал, что критические температуры СП как для CO, так и для AFM-упорядочения качественно воспроизводят численные результаты, но систематически дают более высокие значения. Расчеты методом МК показывают, что случаи сильного и слабого обмена качественно различаются. В случае слабого обмена возникает фрустрация в зарядово-упорядоченном основном состоянии системы. Однородная АFМ-фаза в пределе сильного обмена неустойчива по отношению к ФР псевдоспиновой и спиновой подсистем. Получено аналитическое выражение для температуры ФР, и мы обнаружили, что оно хорошо согласуется с результатами численного моделирования классическим методом МК.

\section{Список литературы}

[1] R.J. Birgeneau, C. Stock, J.M. Tranquada, K. Yamada. J. Phys. Soc. Jpn 75, 111003 (2006).

[2] J.M. Tranquada, G. Xu, I.A. Zaliznyak. J. Magn. Magn. Mater. 350, 148 (2014).

[3] T. Wu, H. Mayaffre, S. Krämer, M. Horvatić, C. Berthier, W.N. Hardy, R. Liang, D.A. Bonn, M.-H. Julien. Nature 477, 191 (2011).

[4] G. Ghiringhelli, M. Le Tacon, M. Minola, S. BlancoCanosa, C. Mazzoli, N.B. Brookes, G.M. De Luca, A. Frano, D.G. Hawthorn, F. He, T. Loew, M. Moretti Sala, D.C. Peets, M. Salluzzo, E. Schierle, R. Sutarto, G.A. Sawatzky, E. Weschke, B. Keimer, L. Braicovich. Science 337, 821 (2012).

[5] J. Chang, E. Blackburn, A.T. Holmes, N.B. Christensen, J. Larsen, J. Mesot, R. Liang, D.A. Bonn, W.N. Hardy, A. Watenphul, M.V. Zimmermann, E.M. Forgan, S.M. Hayden. Nature Phys. 8, 871 (2012).

[6] E.H. da Silva Neto, P. Aynajian, A. Frano, R. Comin, E. Schierle, E. Weschke, A. Gyenis, J. Wen, J. Schneeloch, Z. Xu, S. Ono, G. Gu, M. Le Tacon, A. Yazdani. Science 343, 393 (2014).

[7] R. Comin, A. Damascelli. Ann. Rev. Condens. Matter Phys. 7, 369 (2016).

[8] F. Laliberté, M. Frachet, S. Benhabib, B. Borgnic, T. Loew, J. Porras, M. Le Tacon, B. Keimer, S. Wiedmann, C. Proust, D. LeBoeuf. npj Quantum Mater. 3, 11 (2018).

[9] P. Abbamonte, A. Rusydi, S. Smadici, G.D. Gu, G.A. Sawatzky, D.L. Feng. Nature Phys. 1, 155 (2005).

[10] E. Berg, E. Fradkin, S.A. Kivelson, J.M. Tranquada. New J. Phys. 11, 115004 (2009).

[11] M. Fujita, H. Hiraka, M. Matsuda, M. Matsuura, J.M. Tranquada, S. Wakimoto, G. Xu, K. Yamada. J. Phys. Soc. Jpn 81, 011007 (2012).

[12] E. Fradkin, S.A. Kivelson. Nature Phys. 8, 864 (2012).

[13] G. Drachuck, E. Razzoli, G. Bazalitski, A. Kanigel, C. Niedermayer, M. Shi, A. Keren. Nature Commun. 5, 3390 (2014).

[14] O. Cyr-Choinière, G. Grissonnanche, S. Badoux, J. Day, D.A. Bonn, W.N. Hardy, R. Liang, N. Doiron-Leyraud, L. Taillefer. Phys. Rev. B 92, 224502 (2015).

[15] A.S. Moskvin. Phys. Rev. B 84, 075116 (2011).

[16] A.S. Moskvin. J. Phys.: Condens. Matter 25, 085601 (2013).
[17] Yu.D. Panov, A.S. Moskvin, A.A. Chikov, I.L. Avvakumov. J. Superconductiv. Nov. Magn. 29, 1077 (2016).

[18] Yu.D. Panov, A.S. Moskvin, A.A. Chikov, K.S. Budrin. J. Low Temp. Phys. 187, 646 (2017).

[19] Yu.D. Panov, K.S. Budrin, A.A. Chikov, A.S. Moskvin. Письма в ЖЭТФ 106, 242 (2017).

[20] M. Blume. Phys. Rev. 141, 517 (1966).

[21] H.W. Capel. Physica 32, 966 (1966).

[22] M. Blume, V.J. Emery, R.B. Griffiths. Phys. Rev. A 4, 1071 (1971).

[23] С.В. Сёмкин, В.П. Смагин. ФТТ 57, 926 (2015).

[24] K. Yaldram, G.K. Khalil, A. Sadiq. Solid State Commun. 87, 1045 (1993).

[25] G.K. Khalil, K. Yaldram, A. Sadiq. Int. J. Mod. Phys. C 08, 139 (1997).

[26] K. Kapcia, S. Robaszkiewicz, R. Micnas. J. Phys. Condens. Matter 24, 215601 (2012).

[27] K. Kapcia, S. Robaszkiewicz. J. Phys. Condens. Matter 25, 065603 (2013).

[28] K.J. Kapcia, S. Murawski, W. Klobus, S. Robaszkiewicz. Physica A: 437, 218 (2015).

Редактор Ю.Э. Китаев 